\title{
Fabrication and characterization of meltblown Poly(Vinylidene difluoride) membranes
}

\author{
Salvatore Luiso, James J. Henry, Behnam Pourdeyhimi, Peter S. Fedkiw
}

\section{SUPPORTING INFORMATION}
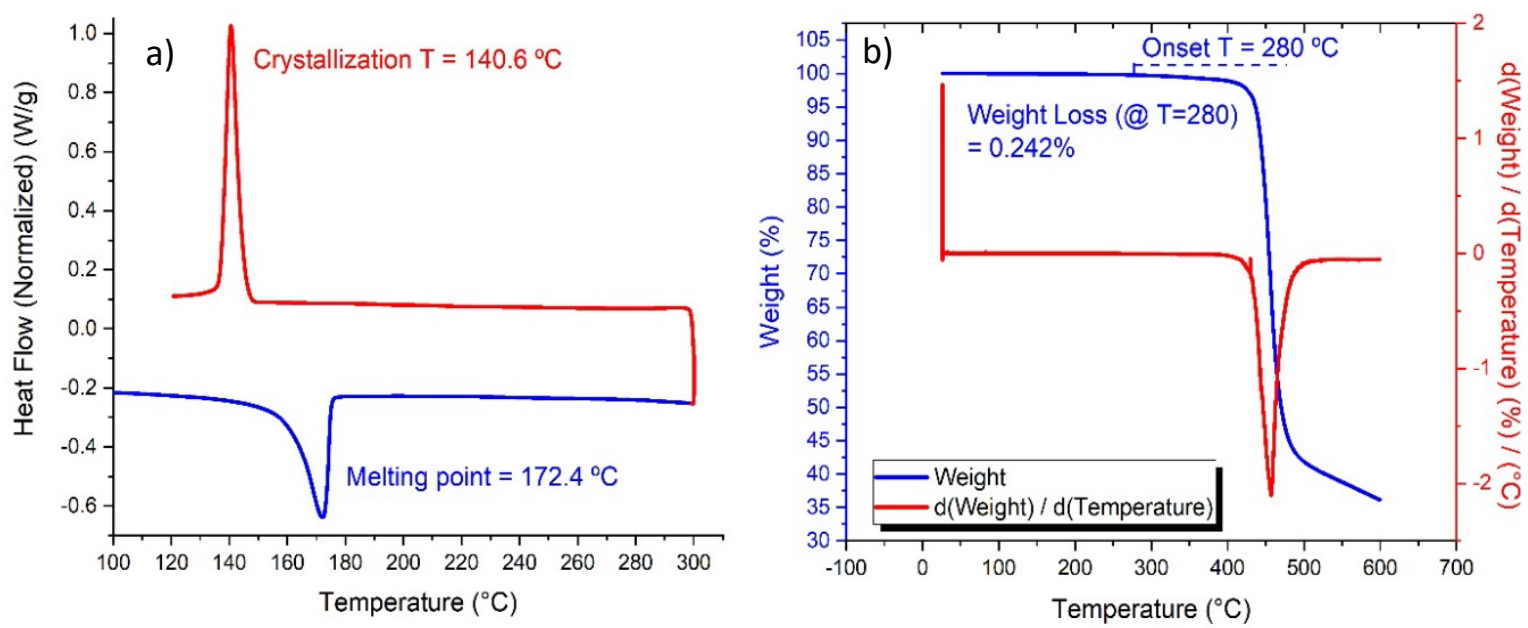

Figure S1. DSC (a) and TGA (b) of PVDF Kynar resin RC 10,287.

\section{RHEOLOGICAL EXPERIMENTS}

Temperatures $=200 / 220 / 240 / 260{ }^{\circ} \mathrm{C}$

1. Steady shear experiment

Shear rate range $=0.01-100 \mathrm{~s}^{-1}$

Geometry: parallel plate

2. Oscillatory experiment

$\%$ strain $=10 \%$

Frequency $(\omega)$ range $=0.01$ to $600 \mathrm{~s}^{-1}$

Geometry: parallel plate

- Cox-Merz rule: $\eta(\omega)=\eta(\dot{\gamma})$

3. Capillary rheometry

Shear rate range $(\mathrm{s}-1)=100-10000$

Geometries (Length:Diameter): 5:1, 20:1, 30:1 
- End pressure effect. We used equation S1 (Bagley corrections) to determine the true shear stress ${ }^{1}$.

$$
\tau=\frac{P}{4(L / D+e)}
$$

where, $\tau$ is the true shear stress, $P$ is the test pressure, $L / D$ is the die length to diameter ratio and $e$ is the die-length correction.

- Non-homogeneous flow. We used equation S2 (Rabinowitz corrections) to determine the true shear rate ${ }^{1}$.

$$
\dot{\gamma}=\frac{\dot{\gamma}_{a p}}{4}\left[3+\frac{d \log \dot{\gamma}_{a p}}{d \log \tau}\right]
$$

where, $\dot{\gamma}$ is the true shear rate, $\dot{\gamma}_{a p}$ is the apparent shear rate, and $d\left(\log \dot{\gamma}_{a p}\right) / d(\log \tau)$ is the slope of the curve $\log \dot{\gamma}_{a p}$ vs $\log \tau$, and $\tau$ is the true shear stress from equation S1.

By superimposing the data from these three experiments we were able to create viscosity curves over a wide range of shear rates, i.e. from 0.01 to $10000 \mathrm{~s}^{-1}$. Figure S1 shows an example of the superimposition.

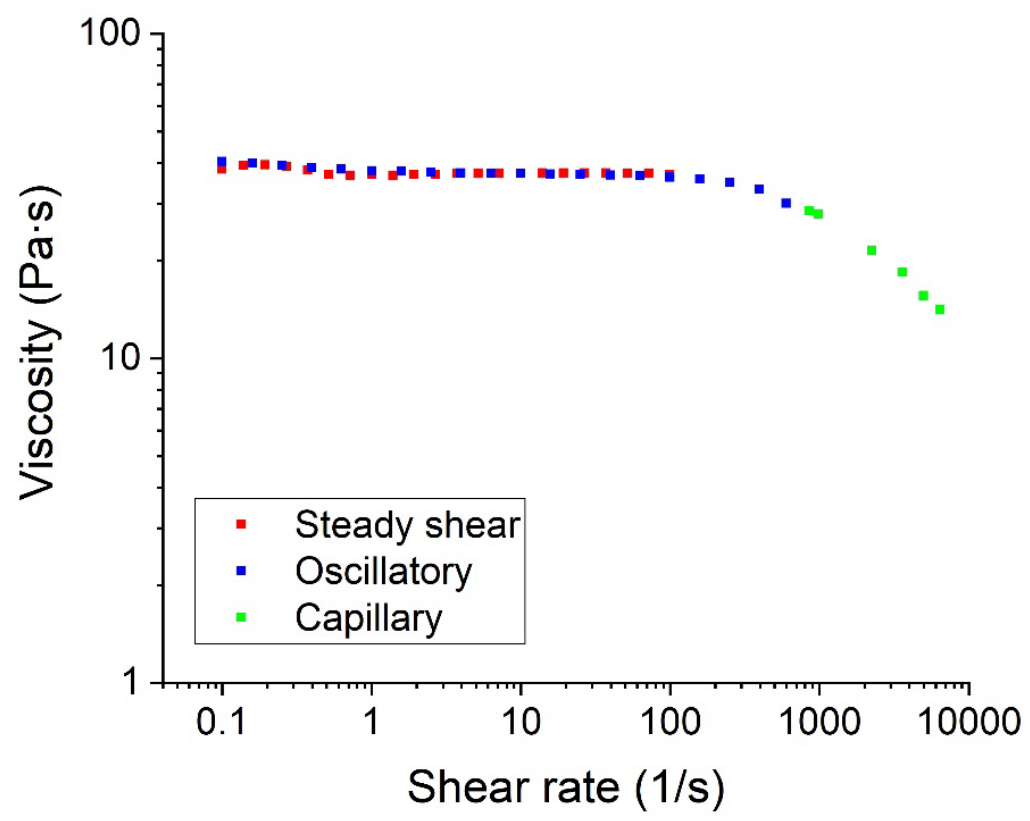

Figure S2 Viscosity curves from steady shear, oscillatory, and capillary rheometry experiments of PVDF Kynar $^{\circledR}$ resin $R C 10,287$ at $200^{\circ}$ C. 


\section{CRYSTALLINITY ACROSS A FIBER CROSS-SECTION}

We soaked both the as-received PVDF pellets and the melt-blown PVDF membranes in 1M LiPF 6 $\mathrm{EC} / \mathrm{DMC}$ battery electrolyte at room temperature for 4 weeks, while continuously stirring the solution. No visible dissolution of the membranes or the polymer pellets was detected. The fibers absorbed electrolyte and did not dissolve at room temperature. By visual inspection, the polymer pellets did not absorb electrolyte. Our explanation for this phenomenon is a change in crystallinity across the fiber cross-section: a highly-crystalline core prevents the fiber from dissolving. Moreover, because the difference in crystallinity between the polymer pellets (53\%) and the fibers $(48 \%)$ is small, the only difference in terms of crystalline and amorphous regions between the polymer pellets and the fibers is the presence of a second population of crystals in the fibers (visible in the DSC experiment). We used electron diffraction TEM to explore the change in crystallinity across the fiber cross-section ${ }^{1}$. We embedded the fiber mat in an epoxy resin and we used Focused Ion Beam etching to make a clean cut of the fiber cross-sections (Fig. S3a). We examined the fiber mats with a Talos F200X TEM, and we used a bar across the fiber cross-sections as a beam-stopper to cover the bright central beam spot so that more diffuse intensity spots could be captured. It was possible to notice a change in the diffraction patterns (in both intensity spots and concentric rings) while moving the bar across the fiber cross-section (Fig. S3 b,c,d), meaning that there was a difference in crystallinity across the cross-section. Unfortunately, the bar was too large to be able to quantify the change in crystallinity.
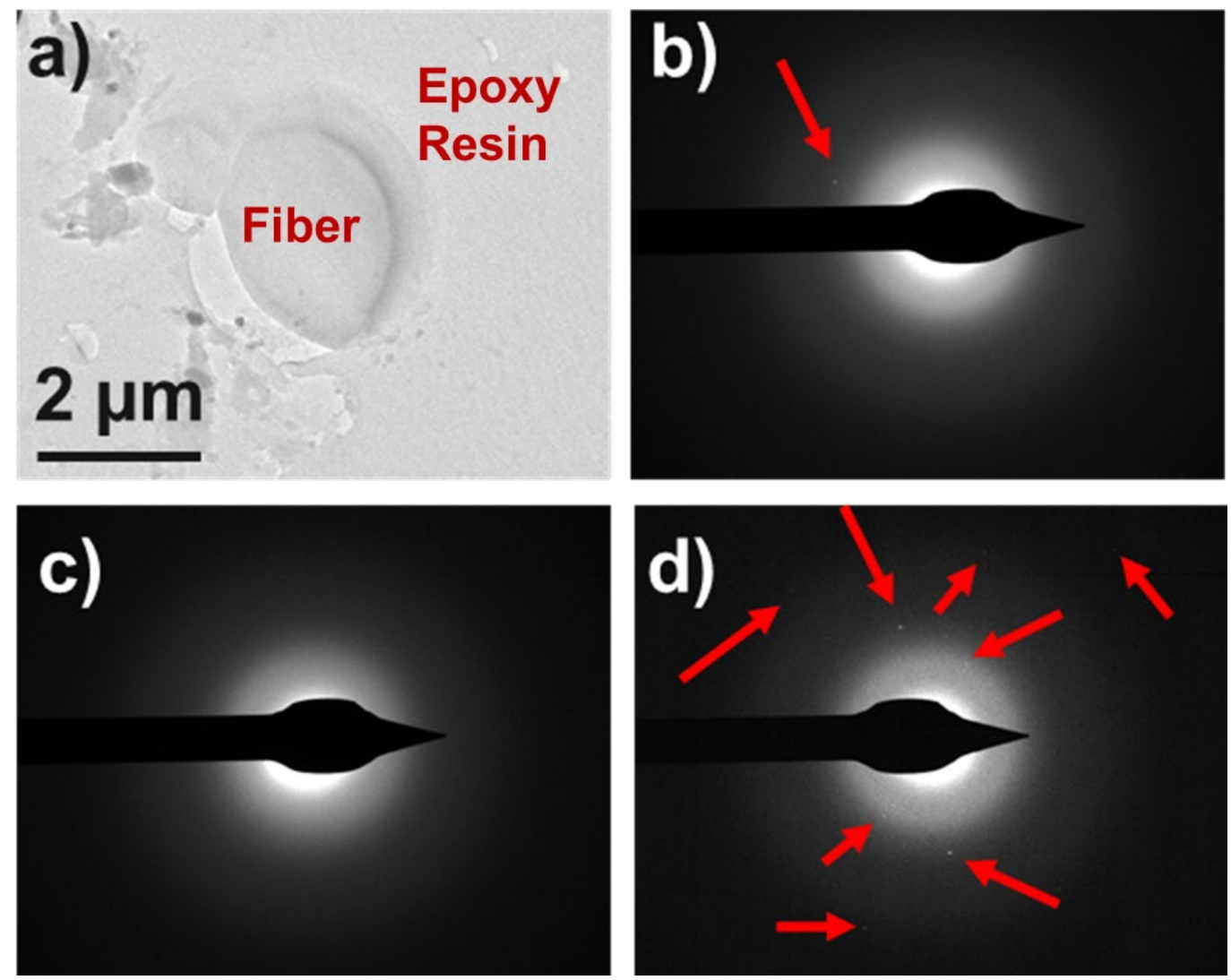

Figure S3 TEM image of fiber cross-section (a), TEM diffraction patterns at different positions of the beam-stopper bar (b), (c), and (d). Red arrows indicate diffraction intensity spot. 
Table S1 Tensile properties of four representative meltblown PVDF samples.

\begin{tabular}{|c|c|c|c|c|}
\hline & \multicolumn{2}{|c|}{ Cross direction } & \multicolumn{2}{c|}{ Machine Direction } \\
\hline Sample & $\begin{array}{c}\text { Peak load } \\
(\mathrm{kPa})\end{array}$ & $\begin{array}{c}\text { Strain at break } \\
(\%)\end{array}$ & $\begin{array}{c}\text { Peak load } \\
(\mathrm{kPa})\end{array}$ & $\begin{array}{c}\text { Strain at break } \\
(\%)\end{array}$ \\
\hline 3 & 0.62 & 11 & 1.52 & 8 \\
\hline 8 & 0.48 & 6 & 0.90 & 6 \\
\hline $12\left(@ 40 \mathrm{~g} \mathrm{~m}^{-2}\right)$ & 1.24 & 11 & 2.28 & 9 \\
\hline 18 & 0.62 & 9 & 1.34 & 6 \\
\hline
\end{tabular}

\section{References}

1. An B.S., Kwon Y., Cha H.W., Kang M.C., Oh J.S., Yang C.W., "Quantification of crystallinity using zero-loss filtered electron diffraction”, Microsc Res Tech., 2019;82:3946. 